\title{
Effects of indirect toxicity of interacting Cd-Zn in a planktonic marine system
}

\author{
Orlando Jara ${ }^{1, *}$, Adriana Aránguiz-Acuña ${ }^{2}$
}

\begin{abstract}
${ }^{1}$ Biología Marina, Facultad de Ecología y Recursos Naturales, Universidad Andres Bello, República 440, Santiago, Chile ${ }^{2}$ Departamento de Química, Facultad de Ciencias, Universidad Católica del Norte, Av. Angamos 0610, Antofagasta, Chile
\end{abstract}

\begin{abstract}
Traditional toxicity bioassays usually assess the effects of single substances on isolated species, which does not, however, allow the identification of potential effects in natural systems. In addition, quantitative estimations of metal uptake in top consumers due to feeding are generally focused on the individual level and do not evaluate the consequences of metal addition at demographic or population levels. An experimental system, composed of the marine microalga Nannochloris oculata Droop, 1955 and the herbivorous rotifer Brachionus plicatilis Müller, 1786, was implemented in order to evaluate (1) whether the interaction of 2 metals, $\mathrm{Cd}$ and $\mathrm{Zn}$, causes a greater toxic effect on planktonic species than that obtained through the addition of single metals and (2) whether the toxicity of these metals on a target herbivore population increases through the indirect route of food intake. Factorial experiments were developed, and results indicated that the toxicity on populations was higher when metals were combined, suggesting resource-dependent interaction. Consumption of contaminated algae had a negative effect on the growth rate of $B$. plicatilis, driven mainly by decreased juvenile and adult survival. This effect was opposite to the direct effect of dissolved metals in the medium. It is suggested that assessment of the interaction between toxic substances, as well as between populations, inserted in trophic networks, is necessary when quantifying the effects of disturbances on marine populations. Finally, our results highlight the relevance of indirect effects using a purely demographic tool focused on population level.
\end{abstract}

KEY WORDS: Metals · Bioassays · Population assessment · Rotifers · Food webs · LTRE · Life table response experiment

\section{INTRODUCTION}

Aquatic pollution is commonly quantified through the evaluation of its impact on biotic components. Pollutants impact aquatic organisms directly, damaging cells and affecting metabolism. This affects performance of individuals and thus their vital rates, which commonly reduces the growth and density of the whole population. Direct effects of contaminants on populations are readily quantified in laboratory toxicity tests, which usually employ model species with exposures to a single toxicant.

Nevertheless, in nature, the toxic effect of pollutants does not act on a single population. Moreover, contaminated habitats usually contain more than a

*Email: orlandoandres.jara@gmail.com single pollutant; these complications might cause unexpected effects. First, indirect effects are produced by interactions among populations. The direct effect of a pollutant on one or more species may be transmitted to other members of the community and spread through the network of ecological interactions (Preston 2002, Fleeger et al. 2003). Therefore, the net effect (sum of all direct and indirect effects) of contamination on a natural population can only be evaluated if the minimum environmental conditions are considered, i.e. dealing with the focal population as part of an interaction network with other populations.

Second, contaminated systems are simultaneously exposed to combinations of stressors including different chemical contaminants. For instance, several

(C) The authors 2013. Open Access under Creative Commons by Attribution Licence. Use, distribution and reproduction are unrestricted. Authors and original publication must be credited. 
heavy metals may be released into the water column from a single contaminating source, or a single released metal can act in combination with other pollutants released from the sediments (Wikfors \& Ukeles 1982, Evtushenko et al. 1984, Cairns \& Buikema 1987, Landis \& Yu 2005). Each single stressor may impact species within a natural system in a particular way, but, in association with other components, may produce synergistic effects (Cassee et al. 1998). Thus, multiple stressors may have an unpredictable effect on the community (Fleeger et al. 2003).

Two common heavy metals that co-occur in nature are cadmium $(\mathrm{Cd})$ and zinc $(\mathrm{Zn})$. Cd is a highly toxic heavy metal (Tortell \& Price 1996, Nassiri et al. 1997), the effects of which may be observed at several trophic levels of marine food webs, including microand macroalgae, zooplankton, molluscs and crustaceans (Elinder \& Piscator 1978, Nassiri et al. 1997, Juárez-Franco et al. 2007). On the other hand, although $\mathrm{Zn}$ is an essential micronutrient for phytoplankton, which is used as a cofactor in many enzymes (Aravind \& Vara 2005, Xu et al. 2012), at high concentrations it may be toxic (Juárez-Franco et al. 2007). Earlier evidence suggested that the combined effect of $\mathrm{Cd}$ and $\mathrm{Zn}$ at high concentrations could be lethal at any trophic level (Elinder \& Piscator 1978, Wolter et al. 1984, Lee \& Morel 1995, Aravind \& Vara 2005, Juárez-Franco et al. 2007). As $\mathrm{Cd}$ is known to replace $\mathrm{Zn}$ for some biological functions in a selection of phytoplankton species (Lane \& Morel 2000, Xu et al. 2012), a certain degree of interaction between these metals is expected.

In the present study an experimental marine planktonic system was created, consisting of the rotifer Brachionus plicatilis (consumer) and the microalga Nannochloris oculata (food resource). Both species are suitable for use in toxicity bioassays because of their sensitivity to several contaminants, easy maintenance in the laboratory and their functional role in aquatic ecosystems (Snell \& Janssen 1995, Marcial et al. 2005, Nandini et al. 2007, Snell \& Joaquim-Justo 2007). In this system we assessed 2 means of metal uptake on rotifer individuals, one direct way defined as uptake of dissolved metals in the medium and another defined as indirect uptake through consumption of food treated with metals in sublethal concentrations. The objectives of this study were (1) to determine the existence of an interaction in the toxicity of $\mathrm{Cd}$ and $\mathrm{Zn}$ and its effect on the growth of rotifer $B$. plicatilis populations and (2) to assess the relevance of direct and indirect metal uptake (dissolved vs. N. oculata ingestion) on the demographic and population response of $B$. plicatilis.

\section{MATERIALS AND METHODS}

\section{Organisms and culture conditions}

The rotifer Brachionus plicatilis and alga Nannochloris oculata were provided by the Marine Research Center of Quintay (CIMARQ), where they have been maintained isolated for $>5 \mathrm{yr}$, under the standard culture conditions used in fish cultures. The marine microalga $N$. oculata was cultured in sterile $F / 2$ medium Guillard (SIGMA) and the rotifer B. plicatilis was cultured in seawater autoclaved at $121^{\circ} \mathrm{C}$, under standardized laboratory conditions. The rotifer stock cultures were fed with $N$. oculata at a density of $0.2 \times$ $10^{5}$ cells $\mathrm{ml}^{-1}$, and the medium was changed every $72 \mathrm{~h}$.

\section{Toxicity on algae}

The toxicity tests of $\mathrm{Cd}\left(\mathrm{NO}_{3}\right)_{2}$ and $\mathrm{Zn}\left(\mathrm{NO}_{3}\right)_{2}$ on Nannochloris oculata were performed at $0,0.01$, $0.1,1.0,10.0$ and $100.0 \mathrm{mg} \mathrm{l}^{-1}$ of the corresponding salt. The assays were conducted for $96 \mathrm{~h}$ in a culture chamber with constant light at $20 \pm 1^{\circ} \mathrm{C}$, with manual agitation every $6 \mathrm{~h}$. $N$. oculata was inoculated in $25 \mathrm{ml}$ medium into $100 \mathrm{ml}$ glass flasks at an initial density of $4.0 \times 10^{4}$ cells $\mathrm{ml}^{-1}$ extracted from stock cultures in log phase growth. From the initial and final densities, the daily growth rate was estimated as the slope of the log-transformed density with time, using least-squares regression. Median effective concentrations (EC50) were estimated, defined as the concentration of trace metals required provoking a response halfway between the control response and the maximum response; here the endpoint was considered as intrinsic population growth (Luoma \& Rainbow 2008). Three concentrations for each metal were selected from the probit curve (corresponding to EC25, EC50 and EC75) to evaluate the effect of a gradient of $\mathrm{Cd}$ and $\mathrm{Zn}$ alone and together on the growth of N. oculata.

In order to control for the addition of nitrates in the treatments, the growth rate of Nannochloris oculata was estimated under a gradient of $\mathrm{NaNO}_{3}$ concentrations. This was performed under identical conditions and with the same concentrations employed in the metal test described above. The difference in the growth rate of $N$. oculata between treatments with and without metal was estimated for each concentration, obtaining the growth rate observed as the metal treatment effect. 


\section{Factorial experiments}

Preliminary acute toxicity tests of $\mathrm{Cd}$ and $\mathrm{Zn}$ on Brachionus plicatilis were performed at 0, 0.0625, $0.125,0.25,0.50$ and $1.0 \mathrm{mg} \mathrm{l}^{-1}$ of the corresponding salt in glass vessels of $50 \mathrm{ml}$, containing 20 female neonates ( $<12 \mathrm{~h}$ old) in $20 \mathrm{ml}$ of seawater. Survival was determined after $24 \mathrm{~h}$. This allows estimation of the percentile lethal concentration (LC) of the metals. After the results obtained in these preliminary tests, concentrations corresponding to LC25, LC50 and LC75 were used for 2 factorial experiments in order to establish a gradient of metal concentrations.

A first 3-way factorial experiment was performed to estimate the combined effect of metal concentration, metal type exposure and food level on rotifer growth rate under laboratory conditions. Levels of the factors were (1) Cd (Cd alone), Zn (Zn alone), and $\mathrm{Cd}-\mathrm{Zn}$ (Cd and $\mathrm{Zn}$ together), for type of metal; (2) control (no metal addition), low level (corresponding to LC25 of each metal), medium level (LC50 of each metal) and high level (LC75 of each metal), for metal concentration; and (3) low $\left(0.05 \times 10^{5}\right.$ cells ml $\left.{ }^{-1}\right)$ and high $\left(0.25 \times 10^{5}\right.$ cells $\left.\mathrm{ml}^{-1}\right)$ Nannochloris oculata density, for food level. These food densities were selected based on previous bioassay studies using $N$. oculata as food (Fernández-Casalderrey et al. 1993). For each treatment, 20 newborns (neonates $<12 \mathrm{~h}$ old) were inoculated in individual $50 \mathrm{ml}$ beakers. Every $24 \mathrm{~h}$, each animal was checked for survival and its offspring were counted and discarded. From these data, age-specific fertility $\left(m_{x}\right.$, computed using the number of live offspring) and age-specific survivorship $\left(s_{X}\right.$, the survival probability from age $x$ to $x+1$ ) were estimated. The experiment was continued until the last female of the cohort died; this allows construction of an age-structured matrix for each experimental combination. The finite population growth rate $(\lambda)$ was calculated as the dominant eigenvalue of the projection matrix. A 3-way ANOVA and a Tukey test for multiple comparisons were conducted in order to identify significant effects of interaction of metal type, concentration and food level on population growth rate.

A second 3-way factorial experiment was performed in order to assess the indirect toxic effect of metals on a Brachionus plicatilis population through the consumption of polluted food. Three experimental factors were used: (1) type of metal, in 3 levels: Cd (Cd alone), Zn (Zn alone) and Cd-Zn (Cd and Zn together); (2) metal source: medium (M, dissolved metal in the medium), alga (A, the metal was incorporated through algae grown in a medium enriched with cadmium and zinc nitrates) and both (M-A); and (3) food level: low $\left(0.05 \times 10^{5}\right.$ cells $\left.\mathrm{ml}^{-1}\right)$ and high $\left(0.25 \times 10^{5}\right.$ cells $\left.\mathrm{ml}^{-1}\right)$ Nannochloris oculata density (i.e. as in the first factorial experiment). For M, concentrations of the salt required by the first factor were $0.24 \mathrm{mg} \mathrm{l}^{-1}$ for $\mathrm{Zn}$ and $0.23 \mathrm{mg} \mathrm{l}^{-1}$ for $\mathrm{Cd}$, obtained from probit regression. For A, microalgae were cultured in a medium with the required metal or metal combination, the concentrations being $0.99 \mathrm{mg} \mathrm{l}^{-1}$ of $\mathrm{Cd}$ and $1.3 \mathrm{mg} \mathrm{l}^{-1}$ of $\mathrm{Zn}$, which are the EC75 estimated above. After 3 d of growth, the culture was centrifuged and cell density was estimated. The rest of the experimental conditions and specifications were the same as in the first factorial experiment. A factorial LTRE analysis (analysis of life table response) with a fixed design was conducted following Caswell (2001) (see Ramos-Jiliberto \& AránguizAcuña 2007). Consequently:

$$
\begin{aligned}
\lambda^{(k l m)} & =\lambda^{(\cdots)}+\alpha^{(k)}+\beta^{(l)}+\gamma^{(m)}+(\alpha \beta)^{(k l)} \\
& +(\beta \gamma)^{(l m)}+(\alpha \gamma)^{(k m)}+(\alpha \beta \gamma)^{(k l m)}
\end{aligned}
$$

where $\lambda\left({ }^{k l m}\right)$ is population growth rate of the matrix obtained with the $k$ th type of metal, the 1 th level of resource and the $m$ th metal concentration. $\alpha\left({ }^{k}\right), \beta\left({ }^{l}\right)$ and $\gamma\left({ }^{m}\right)$ are the main effects of the kth type of metal, the 1 th level of resource and the $m$ th metal concentration, $(\alpha \beta)^{(k l)},(\beta \gamma)^{(l m)}$ and $(\alpha \gamma)^{(k m)}$ are the pairwise interaction effects, and $(\alpha \beta \gamma)^{(k l m)}$ is the 3-way interaction effect. $\lambda^{(\cdots)}$ refers to the population growth rate of the overall mean matrix $(\mathbf{A} \cdots)$. The effect of a factor on the finite growth rate can be further analyzed by estimating the contributions of each matrix coefficient (i.e. the survival and fertility rates):

$$
\begin{gathered}
\alpha^{(k)}=\left.\sum_{i}\left(p_{i}^{(k \cdot)}-p_{i}^{(\cdot)}\right) \frac{\partial \lambda}{\partial p_{i}^{(k \cdot)}}\right|_{P / 2} \\
\beta^{(l)}=\left.\sum_{i}\left(p_{i}^{(\cdot l)}-p_{i}^{(\cdot)}\right) \frac{\partial \lambda}{\partial p_{i}^{(\cdot l)}}\right|_{P / 2} \\
\gamma^{(m)}=\left.\sum_{i}\left(p_{i}^{(\cdot m)}-p_{i}^{(\cdot)}\right) \frac{\partial \lambda}{\partial p_{i}^{(\cdot m)}}\right|_{P / 2} \\
(\alpha \beta)^{(k l)}=\left.\sum_{i}\left(p_{i}^{(k l)}-p_{i}^{(\cdot)}\right) \frac{\partial \lambda}{\partial p_{i}^{(k l)}}\right|_{P / 2}-\alpha^{(k)}-\beta^{(l)} \\
(\beta \gamma)^{(l m)}=\left.\sum_{i}\left(p_{i}^{(l m)}-p_{i}^{(\cdot)}\right) \frac{\partial \lambda}{\partial p_{\dot{(l)}}^{(l m)}}\right|_{P / 2}-\beta^{(l)}-\gamma^{(m)} \\
(\alpha \gamma)^{(k m)}=\left.\sum_{i}\left(p_{i}^{(k m)}-p_{i}^{(\cdot)}\right) \frac{\partial \lambda}{\partial p_{i}^{(k m)}}\right|_{P / 2}-\alpha^{(k)}-\gamma^{(m)} \\
(\alpha \beta \gamma)^{(k l)}=\left.\sum_{i}\left(p_{i}^{(k l m)}-p_{i}^{(\cdot)}\right) \frac{\partial \lambda}{\partial p_{i}^{(k l m)}}\right|_{P / 2}-\alpha^{(k)}-\beta^{(l)}-\gamma^{(m)}
\end{gathered}
$$


where $p_{i}$-values are the vital rate parameters for treatment $k, l$, or $m$, and sensitivities are evaluated at the mean of both parameter sets $(P / 2)$.

An additional data analysis was performed on life tables generated under the the factors food levels and input methods of $\mathrm{Cd}$ and $\mathrm{Zn}$ together (through medium, algae, or both) in order to analyze the effect of source of combined metals on the vital rates. For this analysis, the age-structured matrix was reduced to a stage-structured matrix with 2 stages, juvenile and adult, giving 4 vital rates: juvenile survival $\left(\sigma_{1}\right)$, adult survival $\left(\sigma_{2}\right)$, number of time steps before reaching maturity $(\alpha)$ and adult fertility mean $(\bar{F})$. This data analysis was restricted to the treatment in which rotifers were exposed to both metals together, and was repeated separately for both food levels. The analysis consists of computing rotifer $\lambda$ from a reference matrix $\mathbf{A}(r)$. This $\lambda$ will be referred as $\lambda(r)$. $\mathbf{A}(r)$ is calculated by pooling the data for the 3 treatments for the toxicant source. Based on the vital rates estimated for the $k$ th treatment $(k: 1,2$ and 3, toxicant sources), a finite growth rate $\lambda^{(k)}$ and the difference between $\lambda^{(k)}$ and $\lambda^{(r)}$ are decomposed into contributions due to the effect of the treatment on each vital rate (for detailed estimations see Caswell 1996, Levin et al. 1996, Ramos-Jiliberto \& Aránguiz-Acuña 2007). The equation accounting for this decomposition is:

$$
\lambda^{(k)} \approx \lambda^{(r)}+\left.\sum_{i}\left(p_{i}^{(k)}-p_{i}^{(r)}\right) \frac{\partial \lambda}{\partial p_{i}}\right|_{P / 2}
$$

where the $p_{i}$-values are vital rate parameters of treatment $k$ and reference $r$, and sensitivities $\left(\partial \lambda / \partial p_{i}\right)$ are evaluated at the means of both parameter sets $(P / 2)$.

Confidence intervals (95\%) for $\lambda, \sigma_{1}, \sigma_{2}, \alpha$ and $\bar{F}$ were computed by bootstrap re-sampling with a sample size of 5000 and individual life histories as the unit to be sampled (Meyer et al. 1986, Manly 1997).

In order to detect statistically significant differences between the growth rate in a treatment and the mean growth rate, individual life histories among treatments were permuted according to Manly (1997), with 5000 randomizations. We calculated the statistic $\theta_{i}$ after each permutation; according to Levin et al. (1996), for the $i$ th permutation this is:

$$
\theta_{i}=\left|\lambda_{i}^{(k)}-\lambda^{(k)}\right|
$$

If the observed $\theta$-value was outside $95 \%$ of the distribution of $\theta_{i}$, a significant difference between growth rates was accepted.

Additionally, we quantified the LT50 (see Newman 2001); differences between treatments were tested by the Kruskal-Wallis test.

\section{Consumption experiment}

In order to evaluate whether the presence of the pollutant in the alga modified consumption by Brachionus plicatilis, 20 juvenile individuals were inoculated in $50 \mathrm{ml}$ glass beakers with $0.25 \times 10^{5}$ cells $\mathrm{ml}^{-1}$ of Nannochloris oculata. Algae were cultured at the highest concentrations tested for each metal (see 'Toxicity on algae'). A negative culture was maintained under identical conditions, except that rotifers were absent. Algae in a sample of each culture were counted in a Neubauer chamber $24 \mathrm{~h}$ after the beginning of the experiment. The clearance rate (CR) and the ingestion rate (IR) were estimated as:

$$
\begin{aligned}
\mathrm{CR} & =\frac{\left[\operatorname{Ln}\left(A_{0}-A_{t}\right)\right] \times V}{N \times \Delta t} \\
\mathrm{IR} & =\mathrm{CR} \times \sqrt{A_{0} \times A_{t}}
\end{aligned}
$$

where $A_{0}$ and $A_{t}$ are algal densities at the beginning and end of the experiment, respectively, $\Delta t$ is experiment duration, $V$ is medium volume and $N$ is rotifer density (Soares et al. 2010). Differences in IR between different metal concentrations were tested with 1-way ANOVA.

\section{RESULTS}

The EC and LC values obtained from the Nannochloris oculata and Brachionus plicatilis bioassays show a similar concentration among metals (Table 1). There were no significant differences in EC and LC values between metals ( $t$-test, $\mathrm{p}>0.05$ ).

There were significant effects of food, concentration and type of metal and interaction of paired factors on population growth rates of Brachionus plicatilis (factorial ANOVA $\mathrm{p}<0.05$; Fig. 1). Growth rates were lower when the rotifer was exposed to $\mathrm{Cd}, \mathrm{Zn}$ and $\mathrm{Cd}-\mathrm{Zn}$ than in the control, but the combination of elements generated the lowest $\lambda$ values at both low and high food levels. Significant interaction between metal type and food level was reflected by a decrease in growth rate when $\mathrm{Cd}-\mathrm{Zn}$ was provided at a low food density, contrary to the results observed with single metals. In conclusion, addition of single metals had negative effects on $B$. plicatilis growth when they were incorporated at low concentrations and the food density was low, and slightly negative or null effects at higher concentrations with a high food density. Nevertheless, this pattern was reversed when both metals were incorporated together, producing negative effects 
Table 1. Nannochloris oculata, Brachionus plicatilis. Effective (EC) and lethal (LC) concentrations of $\mathrm{Cd}$ and $\mathrm{Zn}$ obtained from bioassays

\begin{tabular}{|lccccc|}
\hline & \multicolumn{2}{c}{$\mathrm{Cd}$} & \multicolumn{2}{c|}{ Zn } \\
& $\mathrm{mg} \mathrm{l}^{-1}$ & $\mu \mathrm{M}$ & $\mathrm{mg} \mathrm{l}^{-1}$ & $\mu \mathrm{M}$ \\
\hline Nannochloris oculata & & & & \\
Low level (EC25) & 0.33 & 1395.2 & 0.43 & 2270.3 \\
Medium level (EC50) & 0.66 & 2791.3 & 0.87 & 4593.6 \\
High level (EC75) & 0.99 & 4187.5 & 1.3 & 6863.9 \\
Brachionus plicatilis & & & & \\
Low level (LC25) & 0.08 & 338.8 & 0.08 & 422.9 \\
Medium level (LC50) & 0.15 & 634.6 & 0.16 & 844.7 \\
High level (LC75) & 0.23 & 972.4 & 0.24 & 1267.6 \\
\hline
\end{tabular}

at higher concentrations, which were even greater with high resources.

Results of the second factorial experiment showed that population growth rates of Brachionus plicatilis were greater than $\lambda$ of the reference matrix, when metal inputs, either separately or together, were dissolved in the medium. Reduced growth rates were observed when rotifers were exposed to metals through treated algae, and the lowest growth rates were obtained with treated medium and algae. The decrease was greater, in general, with low food density (Fig. 2).

To facilitate interpretation of the LTRE analyses, only single-factor contributions and the contribution of the 3-factor interaction are shown. The factorial LTRE results indicate that the $\mathrm{Cd}-\mathrm{Zn}$ combination treatment generated the greatest (and the only negative) effect on the variation of $\lambda$ for Brachionus pli-

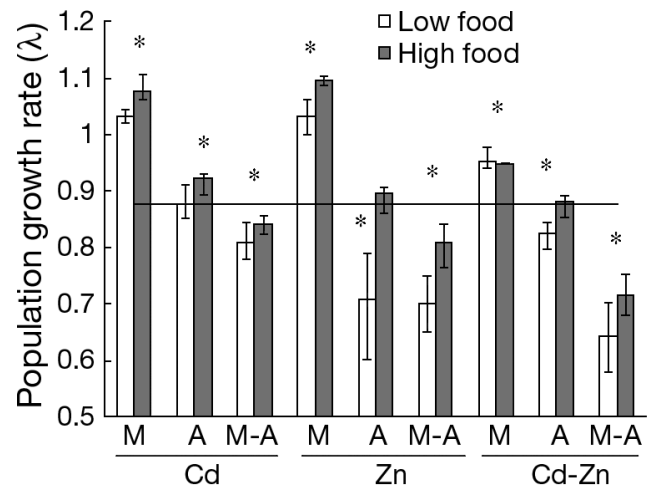

Fig. 2. Brachionus plicatilis. Population growth rates of $B$. plicatilis exposed to metals $(\mathrm{Cd}, \mathrm{Zn}$, or both together) introduced by different routes (medium [M], food [A], or both [MA]) at low $\left(0.05 \times 10^{5}\right.$ cells ml-1) or high $\left(0.25 \times 10^{5} \mathrm{cells} \mathrm{ml}^{-1}\right)$ Nannochloris oculata density. The horizontal line corresponds to the $\lambda$ value of the reference matrix. Error bars show $95 \%$ bootstrap confidence intervals. Significant differences relative to the reference $\lambda$ are shown by asterisks

catilis with respect to the overall mean, while both metals alone produced positive effects on $\lambda$. The positive effect was slightly greater with Cd (Fig. 3a). Fig. 3b shows that resource density generated a marginal contribution to the variation of $\lambda$, with a negative effect of low resources and a positive effect of high resources. The way in which $B$. plicatilis incorporated metal generated the greatest contributions, with positive values occurring when the metal was dissolved in the medium and null contribution when metals were incorporated only through food uptake; the contribution was negative on the rotifer growth rate when the metal was incorporated through the

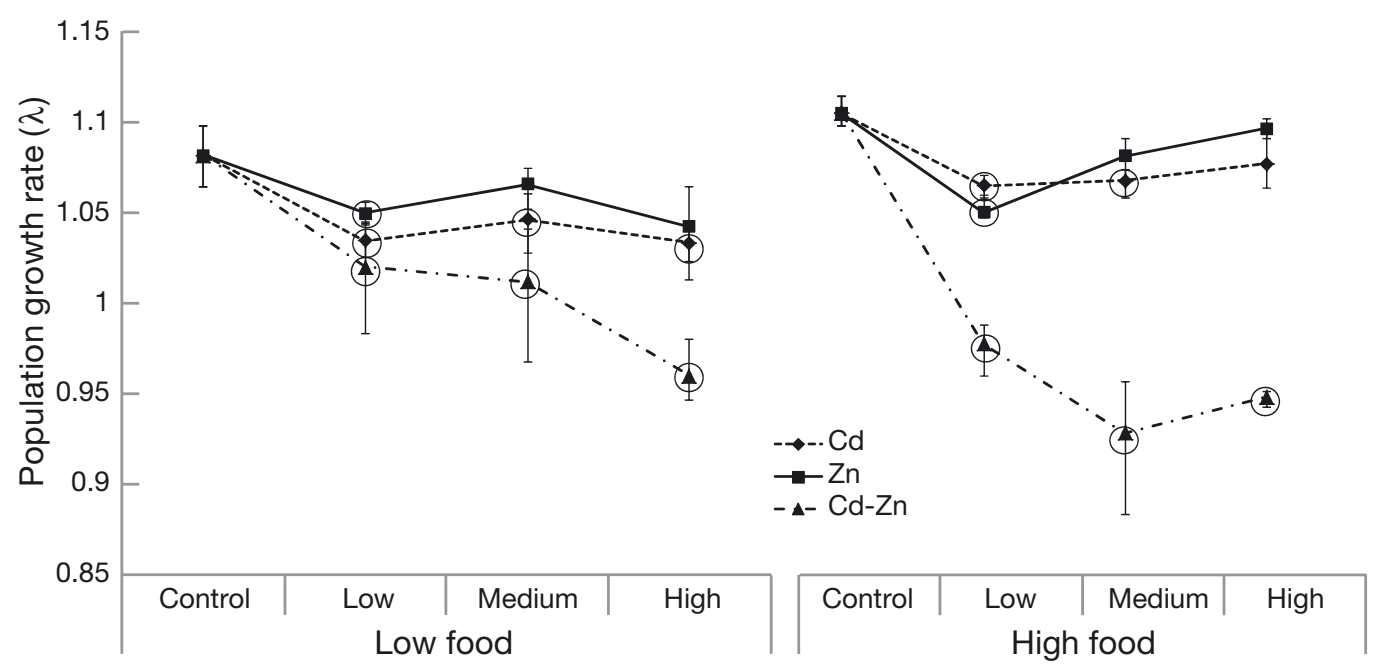

Fig. 1. Brachionus plicatilis. Population growth rates of B. plicatilis exposed to low, medium and high levels of Cd, Zn (see Table 1), or both together, at low $\left(0.05 \times 10^{5}\right.$ cells ml $\left.^{-1}\right)$ or high $\left(0.25 \times 10^{5}\right.$ cells ml $\left.{ }^{-1}\right)$ food densities (Nannochloris oculata). Error bars show $95 \%$ bootstrap confidence intervals. Significant differences between concentrations and between metal types for a given resource level are shown as circles 

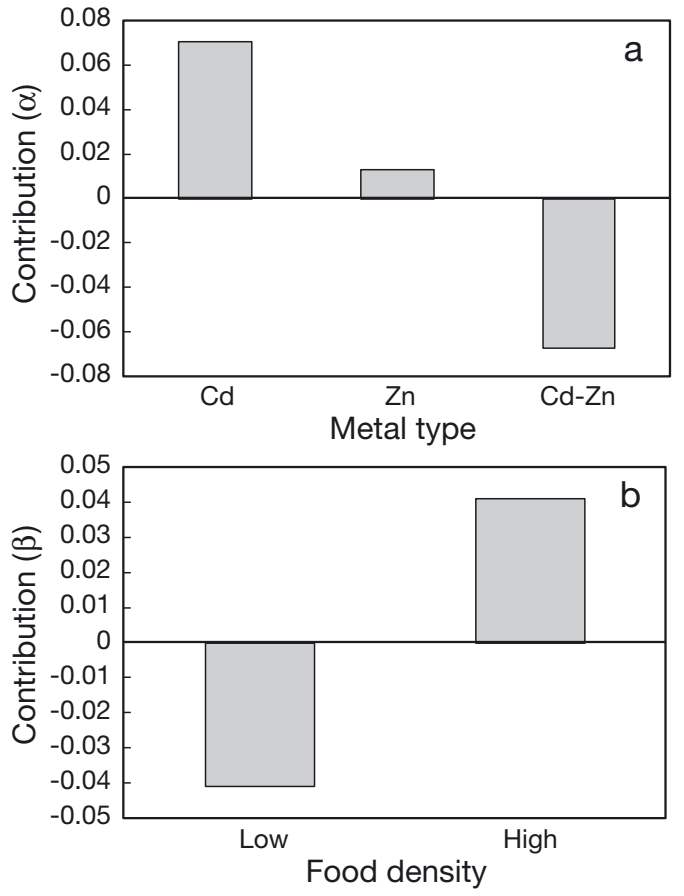

medium and by feeding (Fig. 3c). Finally, the 3 -factor interaction (Fig. 4) showed that the addition of dissolved Cd contributed negatively with both low and high food density; with low food, indirect input (Treatment A) and both inputs (Treatment M-A) contributed positively, but, with high food density, all input forms had negative effects on population growth. Addition of dissolved $\mathrm{Zn}$ to the medium contributed positively to the growth rate of B. plicatilis, at a similar magnitude with both low and high resources. Conversely, the indirect input of Zn through

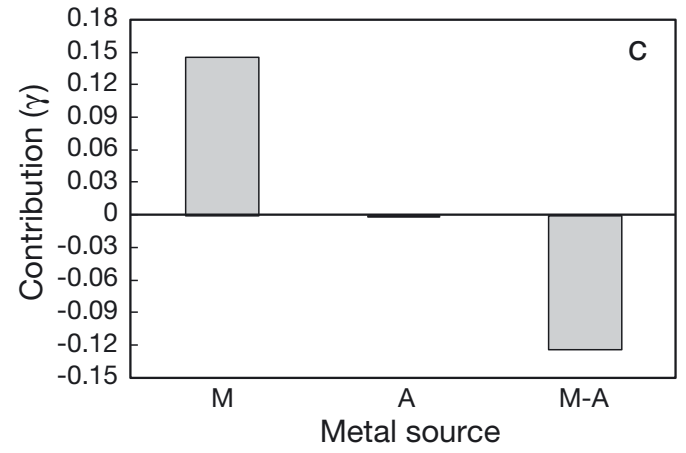

Fig. 3. Brachionus plicatilis. Contributions by life table response experiments of single factors to variation in population growth rate from a reference $\lambda$. (a) Metal type: Cd, Zn, or Cd-Zn, (b) food density: low $\left(0.05 \times 10^{5}\right.$ cells ml ${ }^{-1}$ of Nannochloris oculata) or high $\left(0.25 \times 10^{5}\right.$ cells ml $\mathrm{m}^{-1}$ of $N$. oculata $)$ and (c) metal source: medium (M), algae (A), or both (M-A)

polluted algae generated the largest negative contribution to $\lambda$ with low food density; this contribution was smaller but equally negative with high food density. The net effect generated by the compensation of the 2 input methods was slightly negative with low food and positive with high food. Cd-Zn treatment via the medium generated negative effects on B. plicatilis $\lambda$ at both food levels (slight with low and greater with a high level); the indirect input (Treatment A) contributed positively to the deviation of the additive model, being greater at high food density,
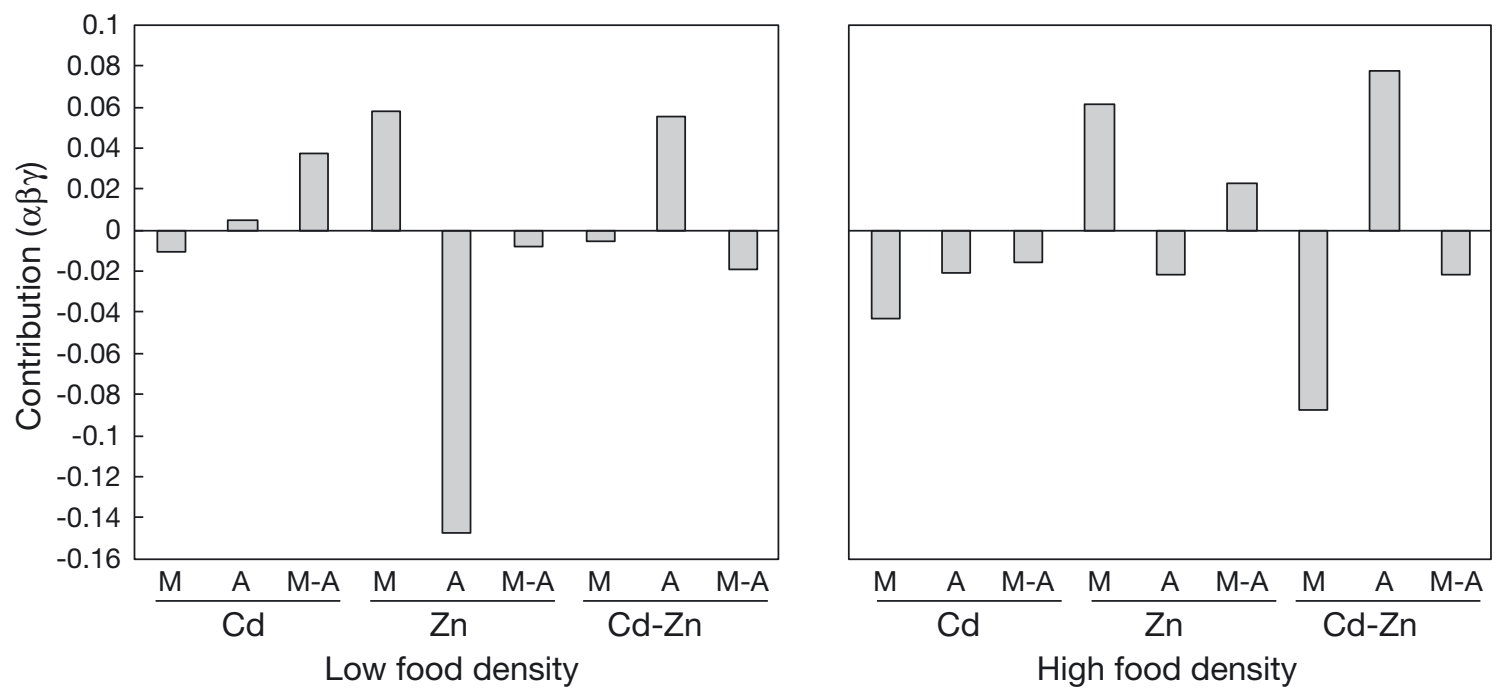

Fig. 4. Brachionus plicatilis. Life table response experiment contributions of interaction effects of metal type/metal source/food density to variation in population growth rate from a reference $\lambda$ 

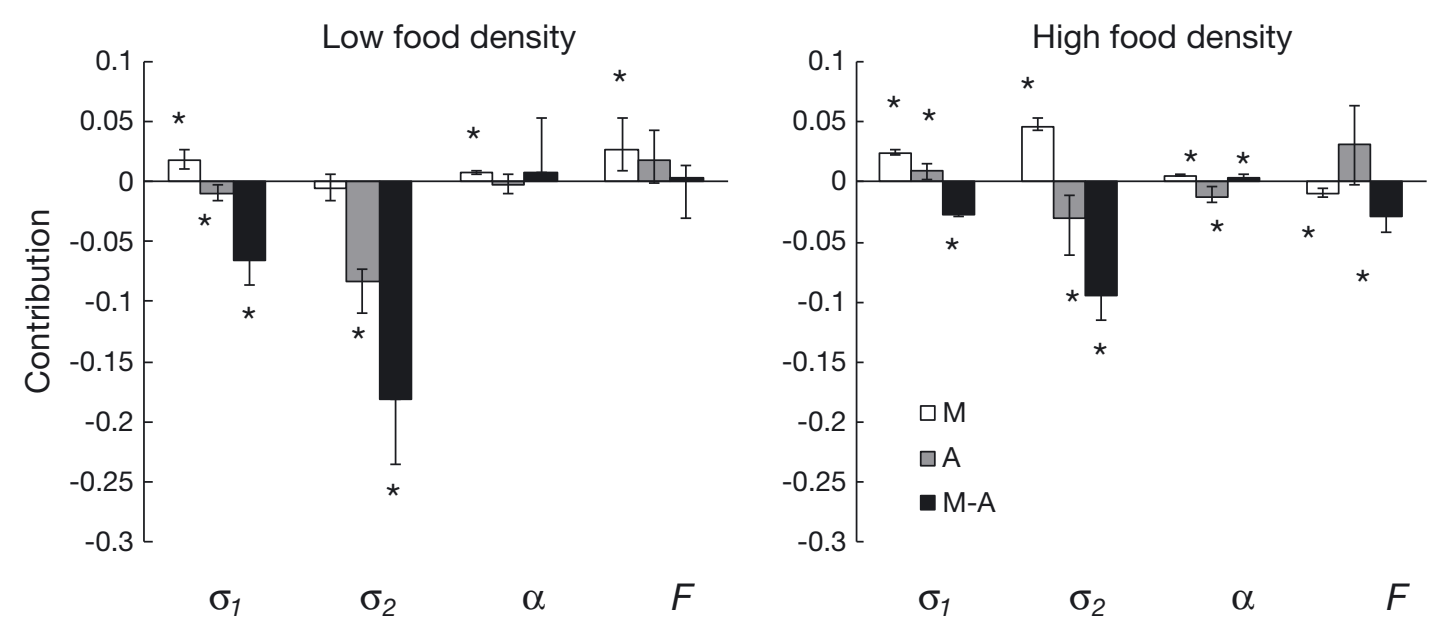

Fig. 5. Brachionus plicatilis. Contribution of demographic parameters: juvenile survivorship $\left(\sigma_{1}\right)$, adult survivorship $\left(\sigma_{2}\right)$, age at maturity $(\alpha)$ and fertility $(F)$ to changes in the growth rate, in response to input of metals through medium filtration (M), feeding with treated algae $(\mathrm{A})$, or both $(\mathrm{M}-\mathrm{A})$. Measurements were obtained at low $\left(0.05 \times 10^{5} \mathrm{cells} \mathrm{ml}^{-1}\right)$ and high $\left(0.25 \times 10^{5} \mathrm{cells}\right.$ $\mathrm{ml}^{-1}$ ) food density levels. Error bars show $95 \%$ bootstrap confidence intervals. Asterisks show significant contributions (different from zero)

and the effect of both inputs via M-A was negative on $B$. plicatilis $\lambda$ with respect to the overall mean. Finally, interaction of all factors showed that the pattern of the form of metal input, either through filtration of the medium or feeding, generated contrary effects. The net effect of the M-A treatment was, in general, compensated by 2 partial vias. The greater negative contribution of $\mathrm{Zn}$ at low food density was compensated for by slight contributions of $\mathrm{Cd}$ addition, while, at the high food level, the negative effect of metals when combined was greater than that obtained with single metals.

The results of 1-way LTRE on the input method of Cd-Zn allowed decomposition of the effects of each treatment on $\lambda$ through the contribution of specific vital rates to the variation of the estimated population growth relative to the overall mean $\lambda$. Fig. 5 shows that, with low food level, the input of dissolved CdZn through the medium (Treatment $M$ ) had marginally positive contributions to $\sigma_{1}, \alpha$ and $\bar{F}$ and higher positive contributions to $\sigma_{1}$ and $\sigma_{2}$ with high food level; there were no negative effects at low food level, and at high food $\bar{F}$ contributed negatively. Food uptake (Treatment A) produced only significant negative contributions of $\sigma_{1}$ and $\sigma_{2}$ when food was at low concentration, while at higher concentration the contribution of $\sigma_{1}$ was positive, and $\sigma_{2}$ and $\alpha$ contributed negatively. The input through both medium and food uptake (Treatment M-A) showed the greatest contributions to $\lambda$ variation, most of them negative: $\sigma_{1}$ and $\sigma_{2}$ with low food level and $\sigma_{1}, \sigma_{2}$ and $\bar{F}$ with high food density. In conclusion, more significant contributions of vital rates to growth rate variation were observed at high food density, but the magnitude of these contributions was greater at low food level. Larger contributions to the decrease in $\lambda$ were observed at the low food level, driven mainly by stronger negative effects on the survival of juveniles and adults when the metal combination was introduced through consumed algae and the medium. Treatment M-A caused the largest negative contributions of all the parameters to the change in $\lambda$, with exception of the slightly positive contribution of $\alpha$ observed at high food density.

Estimation of LT50 (Table 2) showed that exposure to $\mathrm{Cd}, \mathrm{Zn}$, or $\mathrm{Cd}-\mathrm{Zn}$ generated earlier mortality in all treatments compared to the control, but the treatment with algae led to a shorter survival time than when the metal was dissolved in the medium. The shortest survival of Brachionus plicatilis was obtained when it was exposed to both algae and metal dissolved in the medium.

Table 2. Brachionus plicatilis. Median lethal times (LT50, d) according to metal: control (non-metal addition), Cd, $\mathrm{Zn}$, or both $(\mathrm{Cd}-\mathrm{Zn})$ and method of exposure: dissolution in the medium (M), algal food (A), or both (M-A). Lower and upper limits (in parentheses) correspond to $95 \%$ confidence intervals

\begin{tabular}{|lccc|}
\hline Control & $\begin{array}{c}10(8.6-12.7) \\
\mathrm{M}\end{array}$ & $\mathrm{A}$ & $\mathrm{M}-\mathrm{A}$ \\
\hline $\mathrm{Cd}$ & $7(5.9-9.1)$ & $3(2.5-3.8)$ & $2(1.8-2.9)$ \\
$\mathrm{Zn}$ & $6(4.9-8.2)$ & $3(1.9-3.2)$ & $2(1.6-3.4)$ \\
Cd-Zn & $4(3.4-4.9)$ & $3(1.9-3.8)$ & $2(1.9-2.5)$ \\
\hline
\end{tabular}


There were no significant differences observed in CR or IR between metal concentrations; therefore, it was assumed that Brachionus plicatilis consumption was not dependent on algal quality.

\section{DISCUSSION}

Population growth rates of the algae Nannochloris oculata and the herbivore Brachionus plicatilis were affected after exposure to either Cd or Zn alone, and there was an interactive effect of both metals together. Exposure to single metals affected $B$. plicatilis positively when food density was high, over a range of metal concentrations. Metals incorporated together produced more negative effects with high food resource. These results showed that the increased availability of algae promoted a greater toxicity of the combined metals, which was not observed with single metals.

Analyzing the metal uptake routes, it was observed that the indirect uptake of metals through feeding had a significant contribution to the net toxic effect on Brachionus plicatilis. The toxicity observed was mainly driven by negative contributions of the Cd-Zn interaction, of limited food density and of exposure to metals both dissolved in the medium and incorporated by feeding. In many cases, the direct effect imposed by exposure to a contaminated medium was offset by the effect of feeding with treated algae, particularly with Zn or Cd-Zn. The resultant net effect was explained mainly by a negative influence on juvenile and adult survival which was confirmed by LT50 estimation: $B$. plicatilis indirectly contaminated by feeding died before those exposed directly to the contaminated medium, and this survival was similar to that reached by treatment with medium and food.

Previous studies have shown that bioaccumulation of metals, Cd and Zn among others, plays an important role in their transfer through the diet of many marine consumer organisms (Croteau \& Luoma 2008). Nevertheless, marine organisms also bioaccumulate trace metals from the dissolved phase (Tessier \& Turner 1995). Although it is experimentally difficult to quantify accumulation by ingestion, it has been empirically demonstrated that zooplankton ingest $\mathrm{Cd}$ and Zn from their food (see Fisher \& Reinfelder 1995). The relevance of this uptake route relative to the dissolved uptake route has been explained as follows: elements accumulated from ingested food become more concentrated in internal soft tissues than in surface organs and tissues, with potential consequences on the magnitude and immediacy of the effects of trace metals. Conversely, trace metals that follow dissolved uptake routes are associated with hard structures such as phytoplankton cell walls or zooplankton exoskeletons, which can remove non-assimilated metals by sinking fecal pellets (Fisher \& Reinfelder 1995). Under an individualistic approach it has been shown that most of the $\mathrm{Zn}$ found in copepods is obtained from ingested food, with higher assimilation efficiency at low food concentrations, while most of the $\mathrm{Cd}$ accumulates from the dissolved phase (Wang et al. 1996, Wang \& Fisher 1998). Nevertheless, the relative importance of trace element uptakes from the dissolved phase versus food ingestion in overall metal uptake depends on many specific consumer-resource traits and environmental conditions, making it difficult to determine general patterns and to predict effects (Langston \& Spence 1995, $\mathrm{Xu}$ et al. 2012).

Our results allow us to confirm that an indirect effect due to feeding herbivores with algae grown under exposure to metals is a major component in the net toxic effect of metals on herbivores, even though the rotifers were growing in clean standard medium. Moreover, in our experimental design, metal concentrations in algae that served as food were lower than the highest concentration imposed directly on the rotifer, and this showed greater toxic effects on its demographic parameters. These results suggest that in the transfer process from a basal trophic level towards higher trophic levels toxicity may be amplified by bioaccumulation. Therefore, if food density available in the environment can modify the observed total toxic effect at the population level, eutrophic environments may be especially vulnerable to the discharge of inorganic substances such as metals, with interactive negative effects on consumer populations. This is particularly because the $\mathrm{Zn}$ and $\mathrm{Cd}$ requirements of the organisms or the uptake of the metals may be indirectly affected by detrimental environmental conditions caused by an increase of organic matter (Xu et al. 2012).

To the best of our knowledge, these results are novel in highlighting the relevance of indirect effects using a purely demographic tool focused on the population level that, although it does not reveal the underlying biochemical mechanisms that produce the observed population change, revealed higher order effects emerging only at the community level. Although the use of the intrinsic population growth rate (or finite population growth rate, as in this study) as the toxicological endpoint has been questioned in some cases (Snell \& Janssen 1995), the estimation of 
the contributions of vital rates $(\sigma 1, \sigma 2, \alpha$ and $\bar{F})$ allowed us to explain the demographic cause of the observed effects at the population level. Nevertheless, the particular life cycle of Brachionus plicatilis would allow analysis of other endpoints that could provide additional information about tolerance to metals or to other toxic substances, e.g. resting-egg hatchability as an endpoint for pesticide exposure (Marcial et al. 2005). The approach used in the present study produces useful information about the ecological performance of a target population, measured as realized population growth. This kind of analysis, therefore, is complementary to single-species bioassays focused on individual response.

Acknowledgements. Authors acknowledge logistic support from CIMARQ and CENMA. A.A.A. acknowledges support from Project FONDECYT 3110176. The authors thank A. M. Mora, A. Díaz and J. González-Barrientos, and 2 anonymous reviewers for their suggestions on an earlier version of this work, and M. Serra, for his valuable comments on an advanced version of the manuscript.

\section{LITERATURE CITED}

Aravind P, Vara M (2005) Cadmium-zinc interactions in a hydrophonic system using Ceratophyllum demersum L.: adaptive ecophysiology, biochemistry and molecular toxicology. Braz J Plant Physiol 17:3-20

Cairns J Jr, Buikema AL Jr (1987) Estimating the impact of pulses of mixtures of chemicals upon aquatic organisms. In: Vouk VB, Butler GC, Upton AC, Parke DV, Asher $\mathrm{SC}$ (eds) Methods for assessing the effects of mixtures of chemicals. Scope, p 285-300

Cassee FR, Groten JP, Van Bladeren PJ, Feron VJ (1998) Toxicological evaluation and risk assessment of chemical mixtures. Crit Rev Toxicol 28:73-101

Caswell H (1996) Demography meets ecotoxicology: untangling the population level effects of toxic substances. In: Newman MC, Jagoe CH (eds) Ecotoxiclogy: a hierarchical treatment. Lewis Publisher, Boca Raton, FL, p 255-292

Caswell H (2001) Matrix population models: construction, analysis, and interpretation, 2nd edn. Sinauer Associates, Sunderland, MA

> Croteau MN, Luoma S (2008) A biodynamic understanding of dietborne metal uptake by a freshwater invertebrate. Environ Sci Technol 42:1801-1806

Elinder CG, Piscator M (1978) Cadmium and zinc relationships. Environ Health Perspect 25:129-132

> Evtushenko Z, Lukyanova O, Khristoforova N (1984) Biochemical changes in selected body tissues of the scallop Patinopecten yessoensis under long-term exposure to low Cd concentrations. Mar Ecol Prog Ser 20:165-170

Fernández-Casalderrey A, Ferrando MD, Andreu-Moliner E (1993) Chronic toxicity of methylparathion to the rotifer Brachionus calyciflorus fed on Nannochloris oculata and Chlorella pyrenoidosa. Hydrobiologia 255/256:41-49

Fisher NS, Reinfelder JR (1995) The trophic transfer of metals in marine systems. In: Tessier A, Turner DR (eds)
Metal speciation and bioavailability in aquatic systems. IUPAC series on analytical and physical chemistry of environmental systems, Vol 3. John Wiley \& Sons, New York, NY, p 363-406

> Fleeger JW, Carman K, Nisbet R (2003) Indirect effects of contaminants in aquatic ecosystems. Sci Total Environ 317:207-233

Juárez-Franco M, Sarma S, Nandini S (2007) Effects of cadmium and zinc on the population growth of Brachionus havanaensis (Rotifera: Brachionidae). Environ Sci Health A 42:1489-1493

Landis W, Yu M (2005) Introduction to environmental toxicology: impacts of chemicals upon ecological systems, 3rd edn. CRC Press, Boca Raton, FL

> Lane T, Morel F (2000) A biological function for Cadmium in marine diatoms. Proc Natl Acad Sci 97:4627-4631

Langston WJ, Spence SK (1995) Biological factors involved in metal concentrations observed in aquatic organisms. In: Tessier A, Turner DR (eds) Metal speciation and bioavailability in aquatic systems. IUPAC series on analytical and physical chemistry of environmental systems, Vol 3. John Wiley \& Sons, New York, NY, p 407-478

$>$ Lee J, Morel F (1995) Replacement of zinc by cadmium in marine phytoplankton. Mar Ecol Prog Ser 127:305-309

> Levin L, Caswell H, Bridges T, DiBacco C, Cabrera D, Plaia G (1996) Demographic responses of estuarine polychaetes to pollutants: life table response experiments. Ecol Appl 6:1295-1313

Luoma SN, Rainbow PS (2008) Metal contamination in aquatic environments: science and lateral management. Cambridge University Press, Cambridge

Manly BFJ (1997) Randomization, bootstrap and Monte Carlo methods in biology. CRC Press, Boca Raton, FL

- Marcial H, Hagiwara A, Snell T (2005) Effect of some pesticides on reproduction of rotifer Brachionus plicatilis Muller. Hydrobiologia 546:569-575

Meyer JS, Ingersoll CG, McDonald LL, Boyce MS (1986) Estimating uncertainty in population growth rates: jacknife vs. bootstrap techniques. Ecology 67:1156-1166

Nandini S, Chaparro D, Cardenas S, Sarma S (2007) Population growth of Brachionus macracanthus (Rotifera) in relation to cadmium toxicity: influence of algal (Chlorella vulgaris) density. J Environ Sci Health A 42:1467-1472

Nassiri Y, Wery J, Mansot J, Ginsburger-vogeh T (1997) Cadmium bioaccumulation in Tetraselmis suecica: an electron energy loss spectroscopy (EELS) study. Arch Environ Contam Toxicol 33:156-161

Newman MC (2001) Fundamentals of ecotoxicology. Lewis Publishers, London

> Preston BL (2002) Indirect effects in aquatic ecotoxicology: implications for ecological risk assessment. Environ Manag 29:311-323

> Ramos-Jiliberto R, Aránguiz-Acuña A (2007) Betweenspecies differences in demographic responses to temperature of coexisting cladocerans. Austral Ecol 32:766-774

> Snell TW, Janssen C (1995) Rotifers in ecotoxicology: a review. Hydrobiologia 313/314:231-247

> Snell TW, Joaquim-Justo C (2007) Workshop on rotifers in ecotoxicology. Hydrobiologia 593:227-232

Soares MCS, Lürning M, Huszar V (2010) Responses of the rotifer Brachionus calyciflorus to two tropical toxic cyanobacteria (Cylindrospermopsis raciborskii and Mycrocystis aeroginosa) in pure and mixed diets with green algae. J Plankton Res 32:999-1008

Tessier A, Turner DR (1995) Metal speciation and bioavail- 
ability in aquatic systems. IUPAC series on analytical and physical chemistry of environmental systems, Vol 3. John Wiley \& Sons, New York, NY

Tortell P, Price N (1996) Toxicity and zinc limitation in centric diatoms of the genus Thalassiosira. Mar Ecol Prog Ser 138:245-254

> Wang WX, Fisher NS (1998) Accumulation of trace elements in a marine copepod. Limnol Oceanogr 43:273-283

Wang WX, Reinfelder JR, Lee BG, Fisher NS (1996) Assimilation and regeneration of trace elements by marine copepods. Limnol Oceanogr 41:70-81

Wikfors G, Ukeles R (1982) Growth and adaptation of estu-

Editorial responsibility: Wen-Xiong Wang,

Kowloon, Hong Kong, SAR arine unicellular algae in media with excess copper, cadmium or zinc, and effects of metal-contaminated algal food on Crassostrea virginica larvae. Mar Ecol Prog Ser 7:191-206

Wolter K, Rabsch U, Krischkerl P, Davies A (1984) Influence of low concentrations of cadmium, copper and zinc on phytoplankton of natural water samples. Mar Ecol Prog Ser 19:167-173

$\mathrm{Xu}$ Y, Shi D, Aristilde L, Morel F (2012) The effect of pH on the uptake of zinc and cadmium in marine phytoplankton: possible role of weak complexes. Limnol Oceanogr $57: 293-304$

Submitted: December 12, 2012; Accepted: March 14, 2013 Proofs received from author(s): May 23, 2013 